\title{
AMALFI: Active Monitoring for AtriaL Fibrillation
}

\section{A randomized controlled trial of screening for silent atrial fibrillation in high-risk individuals.}

Ethics Ref:

Date and Version No:

Chief Investigator:

Investigators:
19/LO/0220

2019-04-11 V1.1

Professor Louise Bowman

Professor Barbara Casadei

Dr Nicholas Jones

Dr Christine A'Court

Mr Richard Bulbulia

Mrs Georgina Buck
Sponsor:

Funder:

Chief Investigator

Signature:
University of Oxford

NIHR, Oxford Biomedical Research Centre (BRC)

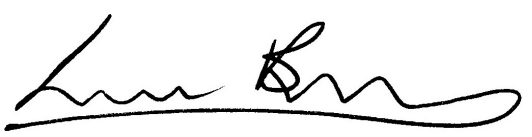

AMALFI Central Coordinating Office, Clinical Trial Service Unit, Richard Doll Building, Old Road Campus, Roosevelt Drive, Oxford OX3 7LF, UK

Tel: Freephone 08081645080 or: +44 (0) 1865743884

Email: amalfi@ndph.ox.ac.uk, website:www.amalfitrial.org 


\section{TABLE OF CONTENTS}

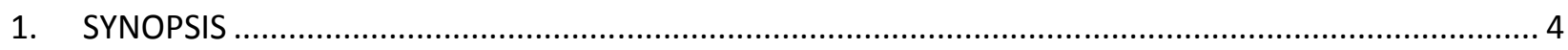

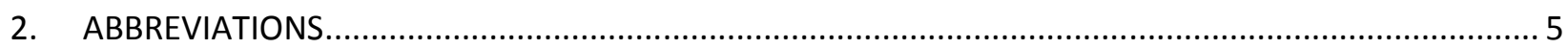

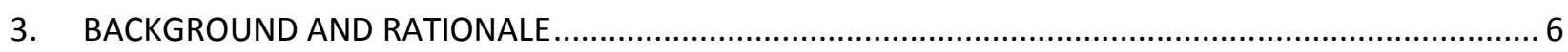

3.1 AF: A global health problem associated with substantial morbidity and mortality ........................... 6

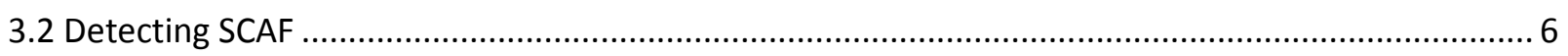

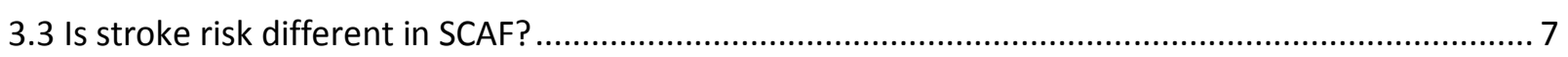

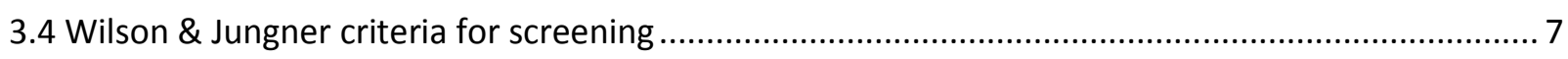

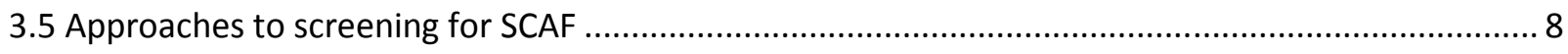

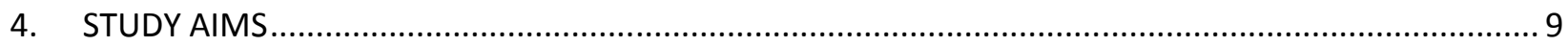

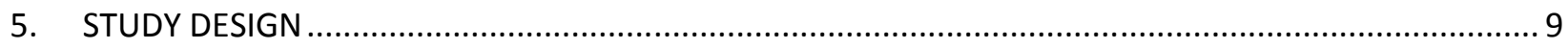

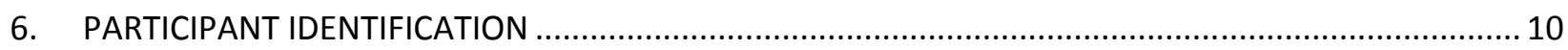

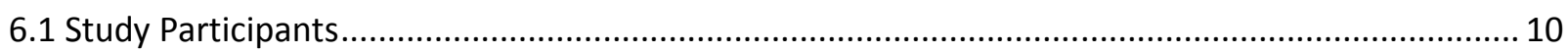

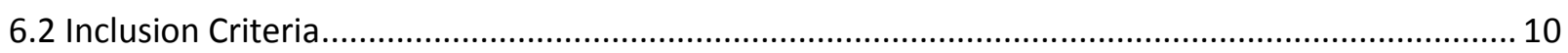

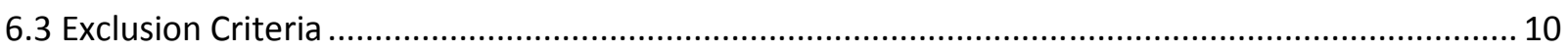

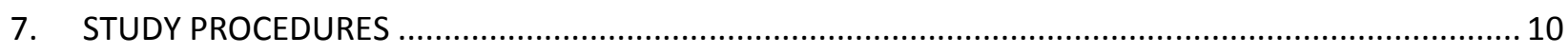

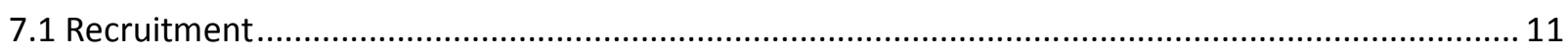

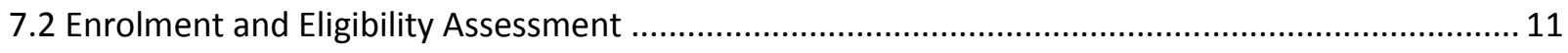

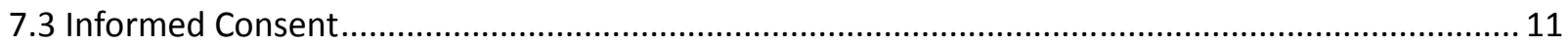

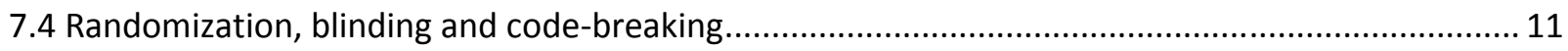

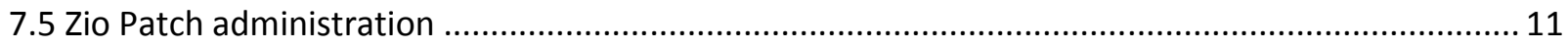

7.6 Discontinuation/Withdrawal of Participants from Study......................................................... 12

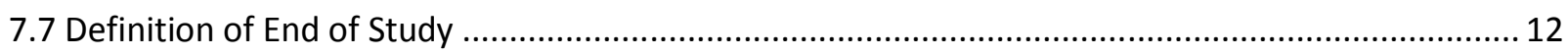

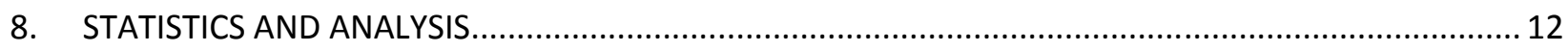

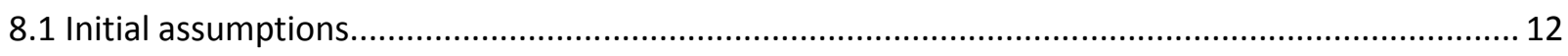

8.2 Sample size and predicted number of diagnoses........................................................................ 13

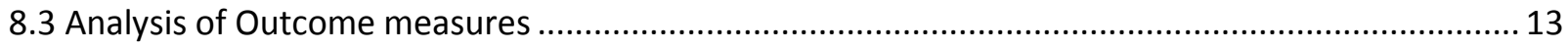

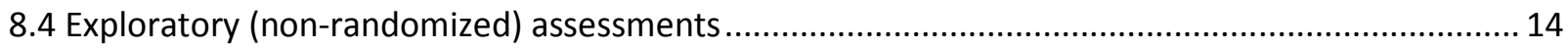

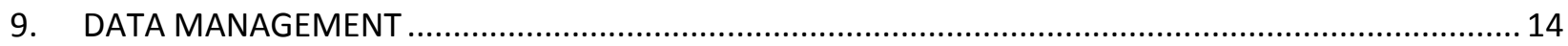

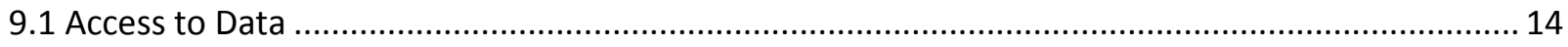

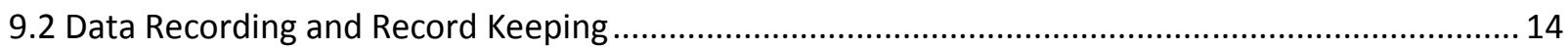

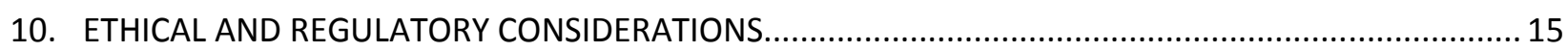

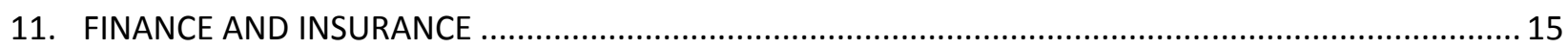

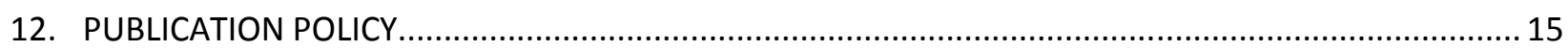




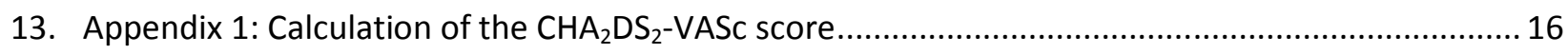

14. Appendix 2: Modelling of expected prevalence of AF over time and statistical power calculations . 17

15. REFERENCES 


\section{SYNOPSIS}

\begin{tabular}{|l|l|}
\hline Study Title & $\begin{array}{l}\text { AMALFI (Active Monitoring for AtriaL Fibrillation): A randomized controlled } \\
\text { trial of screening for silent atrial fibrillation in high-risk individuals. }\end{array}$ \\
\hline Short title & AMALFI \\
\hline Study Design & Randomized controlled trial \\
\hline $\begin{array}{l}\text { Study } \\
\text { Participants }\end{array}$ & $\begin{array}{l}\text { Individuals with a } \mathrm{CHA}_{2} \mathrm{DS}_{2} \text {-VASc score } \geq 3 \text { (men) or } \geq 4 \text { (women), aged } \geq 65 \\
\text { years without known atrial fibrillation (AF) identified from primary care } \\
\text { records. }\end{array}$ \\
\hline $\begin{array}{l}\text { Planned Sample } \\
\text { Size }\end{array}$ & $\begin{array}{l}2500 ; \text { including } 1250 \text { randomized to the Zio Patch and 1250 in the control } \\
\text { arm. }\end{array}$ \\
\hline $\begin{array}{l}\text { Primary } \\
\text { Objective }\end{array}$ & $\begin{array}{l}\text { To assess the effect of } 2 \text { weeks of continuous monitoring using Zio Patch on } \\
\text { the proportion of participants diagnosed with AF compared to usual care at } \\
2.5 \text { years of follow up from randomization (i.e. at around the midpoint of a } \\
\text { proposed 5-year screening interval). }\end{array}$ \\
\hline $\begin{array}{l}\text { Secondary } \\
\text { Objective }\end{array}$ & $\begin{array}{l}\text { To assess the effect of } 2 \text { weeks of continuous monitoring using Zio Patch on } \\
\text { the mean times with AF diagnosed compared to usual care after } 5 \text { years of } \\
\text { follow-up from randomization. }\end{array}$ \\
\hline $\begin{array}{l}\text { Protocol } \\
\text { Number }\end{array}$ & $\begin{array}{l}\text { CTSU_AMALFI 1 } \\
\text { IbctN number }\end{array}$ \\
\hline
\end{tabular}




\section{ABBREVIATIONS}

\begin{tabular}{|l|l|}
\hline AF & Atrial Fibrillation \\
\hline AHRE & Atrial High Rate Episodes \\
\hline CHA $_{2} \mathrm{DS}_{2}$-VASc & Ischaemic stroke risk prediction tool for patients with AF (see Appendix 1) \\
\hline CTSU & Clinical Trial Service Unit \& Epidemiological Studies Unit \\
\hline ECG & Electrocardiogram \\
\hline FDA & Food \& Drug Administration \\
\hline GCP & Good Clinical Practice \\
\hline GP & General Practitioner \\
\hline HRA & Health Research Authority \\
\hline NHS & National Health Service \\
\hline NIHR & National Institute for Health Research \\
\hline SCAF & Silent/sub-clinical AF \\
\hline
\end{tabular}




\section{BACKGROUND AND RATIONALE}

\subsection{AF: A global health problem associated with substantial morbidity and mortality} Atrial fibrillation (AF) is a rapidly growing public health and economic burden. ${ }^{1}$ _ENREF 4 The global number of individuals with clinically diagnosed AF was estimated to be 33 million in 2010 and is expected to double by 2050. ${ }^{1,2}$ Based on clinical cases, 9 million people above the age of 55 years are expected to have AF in Europe alone; this number is predicted to rise to about 18 million by 2060 due to demographic changes and the rising prevalence of risk factors for AF, such as obesity, diabetes and hypertension. ${ }^{3} \mathrm{AF}$ is associated with substantial morbidity and mortality (mostly secondary to an increased risk of stroke), reduced quality of life, and a considerable financial burden. ${ }^{1,2,4}$ In the UK, the cost of caring for patients with AF and its complications in the year 2000 accounted for around 1-2\% of total NHS expenditure. ${ }^{5}$

These figures are likely to underestimate the true prevalence of $A F$, as analysis of prolonged ECG monitoring in patients presenting in sinus rhythm has detected silent/sub-clinical AF (SCAF) episodes in around 6-45\% of subjects, depending on the type and duration of monitoring, the characteristics of the study population (in particular, age and medical history), and the definition of AF. ${ }^{6}$ SCAF may have a significant impact on health and disability ${ }^{7}$ \{Hippisley-Cox, $2008 \# 6141\}$; for example, it may be linked to a significant proportion of strokes of undetermined aetiology (so-called "cryptogenic strokes" which represent approximately $25 \%$ of all ischaemic strokes). ${ }^{8-10}$

\subsection{Detecting SCAF}

A key limiting factor in the detection of SCAF is the short-lasting and infrequent nature of the arrhythmic episodes. ${ }^{11,12}$ One opportunistic ECG recording or pulse palpation will therefore have low specificity and sensitivity for AF diagnosis. A systematic review, including 30 studies and over 122,000 participants, found that the prevalence of SCAF detected by single time-point screening (by ECG or pulse palpation) was modest $(1.0 \%, 95 \% \mathrm{Cl} 0.89-1.04 \%$ in all participants and $1.4 \%(95 \% \mathrm{Cl} 1.2-1.6 \%)$ in those aged $\geq 65) .{ }^{13}$ The STROKESTOP study of screening in 7000 individuals without known AF aged 75-76 detected SCAF in 0.5\% of their cohort on the first ECG. However, with repeated intermittent ECGs (mean 26 per participant) over a two week period, this proportion increased to $3 \%{ }^{14}$

By contrast, long-term ECG monitoring using an implanted device in very high-risk older patients detected a SCAF rate (AF episodes lasting $\geq 5$ minutes) of $34 \%$ per person year $(95 \% \mathrm{Cl}, 28-43 \%$ ). In these very high-risk patients, longer durations of SCAF were also common, with rates of AF $\geq 30$ minutes, $\geq 6$ hours and $\geq 24$ hours being $\sim 22 \%$, $7 \%$, and $\sim 3 \%$ per person year, respectively. ${ }^{11}$ Similarly, in patients with a recent cryptogenic stroke (in whom AF was initially excluded by 12 lead ECG and 24 hours or longer Holter ECG recordings), long-term ECG monitoring using an implanted cardiac monitor detected $A F$ in $8.9 \%$ of participants at 6 months (vs. $1.4 \%$ in those allocated to standard care). ${ }^{10}$ The EMBRACE study found a 5-fold increase in AF detection using a 30-day event recorder compared to conventional ECG monitoring. ${ }^{9}$ ENREF_16

In summary, whilst the reported prevalence of SCAF varies depending on the screening methodology, medical history, and characteristics of the participants, there appears to be a significant proportion of individuals with undetected AF across healthcare systems and for these individuals, stroke may be SCAF's first clinical manifestation. 


\subsection{Is stroke risk different in SCAF?}

Patients with clinically diagnosed AF have a five-fold increase in the risk of ischaemic stroke. Strokes that are related to underlying AF have been associated with greater neurological deficit and an increased risk of permanent disability compared to non-embolic strokes. ${ }^{9,15}$ ENREF_16 Effective prevention of thromboembolic events is available and well-established. Oral anticoagulation with either a vitamin $\mathrm{K}$ antagonist or a direct oral anticoagulant (DOAC), when used appropriately in patients with $\mathrm{AF}$ and a $\mathrm{CHA}_{2} \mathrm{DS}_{2}-\mathrm{VASc}$ score $\geq 2$ (see Appendix 1), can reduce stroke risk by up to $65 \% .{ }^{16} \mathrm{~A}$ key objective in $\mathrm{AF}$ management is therefore to identify patients with AF early and to assess them for oral anticoagulation.

There are limited data to allow a comparison of outcomes in patients with SCAF versus clinically diagnosed AF. A meta-analysis, which included only 2 randomized trials and 4 observational studies (involving 2500 patients with SCAF and 8000 patients with symptomatic AF) found no clear difference in outcomes (and wide confidence intervals) between those with SCAF vs those with symptomatic AF: all-cause death (RR 1.38, 95\% Cl 0.82-2.17); cardiovascular death (RR 0.85, 95\% Cl 0.53-1.36); and stroke/thromboembolism (RR 1.72, (95\% Cl 0.59-5.08). ${ }^{17}$ However, the significant heterogeneity across these studies may account for the lack of difference in outcomes.

Rhythm data recorded by cardiac implanted electronic devices have also been used to assess the relative risk of stroke in patients with device-detected SCAF. In contrast to studies with documented 12-lead ECG evidence of AF, implanted devices may also detect other atrial tachyarrhythmias or be over-sensing and thus they refer to "atrial high rate episodes" (AHRE) with cut-off rates and durations varying between studies. ${ }^{6}$ The ASSERT trial studied 2500 patients aged $\geq 65$ years who had recently had a pacemaker or defibrillator inserted, but with no prior history of AF, and interrogated the devices for AHRE (defined as atrial episodes at a rate of $>190$ beat/min lasting at least 6 minutes) at 3 months after implantation. ${ }^{18}$ After a mean followup of 2.5 years, those found to have subclinical AHRE in the first 3-months had a 2.5-fold increase in the risk of thromboembolic events (HR 2.49, 95\% $\mathrm{Cl} 1.28-4.85$ ) compared to those without AHRE; about half of what would be expected in similar patients with persistent AF. ${ }^{18}$ More recently, the ASSERT investigators have examined the relationship between duration of AHRE episodes and the occurrence of stroke or systemic embolism and found that patients with AHRE duration between 6 minutes and 24 hours were not significantly different from patients without SCAF. Only AHRE episodes $>24$ hours were associated with an increased risk of ischaemic stroke or systemic embolism. ${ }^{19}$

In summary, SCAF may carry a significant thromboembolic risk which appears to be dependent on the SCAF burden and the presence of established risk factors for ischaemic stroke.

\subsection{Wilson \& Jungner criteria for screening}

Screening for AF would adhere to many of the Wilson \& Jungner principles for a screening programme. ${ }^{20}$ SCAF is a common, important and growing health problem, with a suspected prevalence between 1 and 35\% depending on age, risk factors, duration of monitoring and definition of $A F$ in terms of duration of arrhythmia. There is a range of potential approaches to screening, including non-invasive tests with a high degree of acceptability to participants. AF is an important risk factor for stroke and there is an established, highly effective preventative 
treatment for AF-associated thromboembolic events in the form of anticoagulation. Failure to identify and treat AF leaves patients at a considerably higher risk of stroke, disability and death.

There are precedents from other successful screening programmes. For instance, in 2003 the UK established the first national screening programme in the world for diabetic retinopathy. Retinal screening was felt to be important as early stages of the disease were usually asymptomatic and yet diabetic retinopathy was the leading cause of blindness in diabetic adults. Relying on primary care physicians or opportunistic detection of early retinopathy in clinic was proving ineffective as it was technically difficult and not given protected time in routine care. Retinal photography at that time required expensive equipment and highly skilled staff. As a result diabetic retinopathy was typically not diagnosed until late-stage, irreversible disease had developed. Nationwide systematic screening has made the retinal assessment cheap and standardised. ${ }^{21}$ The information obtained from screening now allows the healthcare team to identify individuals who would benefit from early intervention, thereby slowing or preventing disease progression. ${ }^{21,22}$

\subsection{Approaches to screening for SCAF}

SCAF detection presents different challenges, particularly as arrhythmic episodes are short and infrequent and thus potentially difficult to capture. Screening for SCAF has previously been suggested and trialled, although a Cochrane review found only one study met their inclusion criteria. $^{23}$ This study found that both opportunistic screening with pulse palpation and systematic screening with a single ECG increased AF detection over usual care (OR $1.58,95 \% \mathrm{Cl}$ 1.10 - 2.29; and OR 1.57, 95\% Cl 1.08 - 2.26, respectively). ${ }^{23}$ Opportunistic screening appeared to be more cost-effective with an incremental cost per additional case detected of $£ 337$ compared to $£ 1,514$ for systematic screening. ${ }^{24}$ However, opportunistic screening relies on healthcare professionals adding to their usual daily work a task which may often be overlooked. Furthermore, single time-point ECGs can considerably under-estimate the prevalence of SCAF and so a systematic approach with a prolonged period of screening may improve detection rates and reduce the number of subsequent adverse events (e.g. stroke).

More recently the REHEARSE-AF investigators randomized 1000 UK patients $\geq 65$ years old with a CHADS-VASc score $\geq 2$ to twice-weekly ambulatory ECG monitoring (30 seconds of single lead monitoring using the AliveCor Kardia monitor attached to a WiFi-enabled iPod, "iECG") versus routine care. Over the 12-month study period there was a 4-fold increase in AF diagnosis in the iECG group compared with routine care (19 vs 5, HR 3.9, 95\% Cl 1.4-10.4, p=0.007) at a cost of around $£ 8000$ per AF diagnosis. The device was reported to be easy to use and acceptable to this group of patients. ${ }^{25}$

The Zio Patch (iRhythm Technologies, San Francisco CA) is a non-invasive single lead ECG monitoring device. Unlike a Holter monitor, the Zio Patch is discrete and water-resistant, and allows the person wearing it to participate in all routine daily activities with minimal disruption. In a pilot study among about 1600 participants of UK Biobank, acceptability was good and the median wear time was 12.7 days with a median analysable ECG time of $99.1 \%$. iRhythm Technologies have developed and validated an automated system that processes and analyses the ECG data (the ZEUS system) using beat-by-beat QRS detection and a sophisticated analysis algorithm to detect rhythm disturbances such as AF and atrial flutter, their rate, frequency and duration and the total AF burden. The output of the automated analysis is checked by a trained operator and a detailed report is provided to the GP by mail. FDA approval has been received to 
use the Zio Patch and the ZEUS algorithm in clinical practice, and the system was launched in the UK in October 2014. The device has been subject to NICE evaluation. ${ }^{26}$

\section{STUDY AIMS}

The AMALFI trial aims to determine the effect of 2 weeks of continuous non-invasive ECG monitoring using the Zio Patch compared to usual care on rates and time diagnosed with AF over a follow up period of 5 years among high-risk individuals. Practice guidelines recommend oral anticoagulation based on the $\mathrm{CHA}_{2} \mathrm{DS}_{2}$-VASc score (see Appendix 1) and independently of AF burden. ${ }^{27,28}$ By targeting the screening to those older than 65 years and with a $\mathrm{CHA}_{2} \mathrm{DS}_{2}-\mathrm{VASC}$ score $\geq 3$ (men) or $\geq 4$ (women) then the subsequent management of those individuals who are identified with SCAF can be directly informed by current guidelines. ${ }^{28}$

The primary outcome is diagnosis of AF (as recorded in the electronic GP record) by 2.5 years of follow up from randomization (i.e. at around the midpoint of a proposed 5-year screening interval).

In a secondary outcome, AMALFI will assess the effect of the monitoring on the times with AF diagnosed (as above) by $\underline{5 \text { years }}$ of follow-up.

\section{STUDY DESIGN}

AMALFI is a randomized controlled trial comparing a single 2-week screening period for AF with the Zio Patch to usual care in individuals with no history of AF and elevated risk of stroke, based on the $\mathrm{CHA}_{2} \mathrm{DS}_{2}$-VASc score (see Appendix 1). Eligible participants will be identified via an electronic search of GP practice data, approached by mail by their GP and offered the opportunity to participate. In total about 2500 participants will be randomized to Zio Patch or usual care and will be followed-up for an average of about 25 years (Figure 1), initially via their GP records for the primary and secondary outcomes and then via NHS Digital only. A preliminary feasibility study will be conducted to identify and resolve potential practical issues, e.g. difficulties with patients self-administering devices.

Participants in the Zio Patch arm will be sent a barcoded Zio Patch to self-administer, ${ }^{29}$ which will be returned by post directly to the UK iRhythm office for processing before the data is sent to iRhythm's laboratories in the USA for analysis. A report detailing any AF episodes and burden over the duration of ECG recording will be sent by iRhythm to the Central Coordinating Office, CTSU, who will be able to link the report to the individual participant via the patch barcode. The study team will then provide the report to the participant's GP. The study team will not directly initiate treatment or act on the information from the Zio Patch data. Instead, an ECG report and cover letter will be sent back to the participant's GP. The subsequent management will be at the discretion of the GP, allowing the doctor and participant to discuss the management strategy, including anticoagulation, independently.

Relevant outcome data from the electronic health record (including both GP-held data and centrally-held data from NHS Digital) data will be collected at 1 year*, at 2.5 years from

\footnotetext{
* Although the primary and secondary outcomes will be based on data collected at 2.5 and 5 years respectively from randomization, a preliminary data collection will be undertaken at 1 year to ensure that full follow-up information is available for participants who move to a different GP practice or who die prior to the 2.5 year data collection.
} 
randomization (for analysis of the primary outcome) and at 5 years from randomization (for analysis of the secondary outcome). Data items will include documentation of AF diagnosis in the GP record, medication use (including anticoagulants) and hospital attendance and admission information. Further follow up, via NHS Digital central records, will then take place at intervals over the following 20 years.

\section{Figure 1: Outline of trial design}

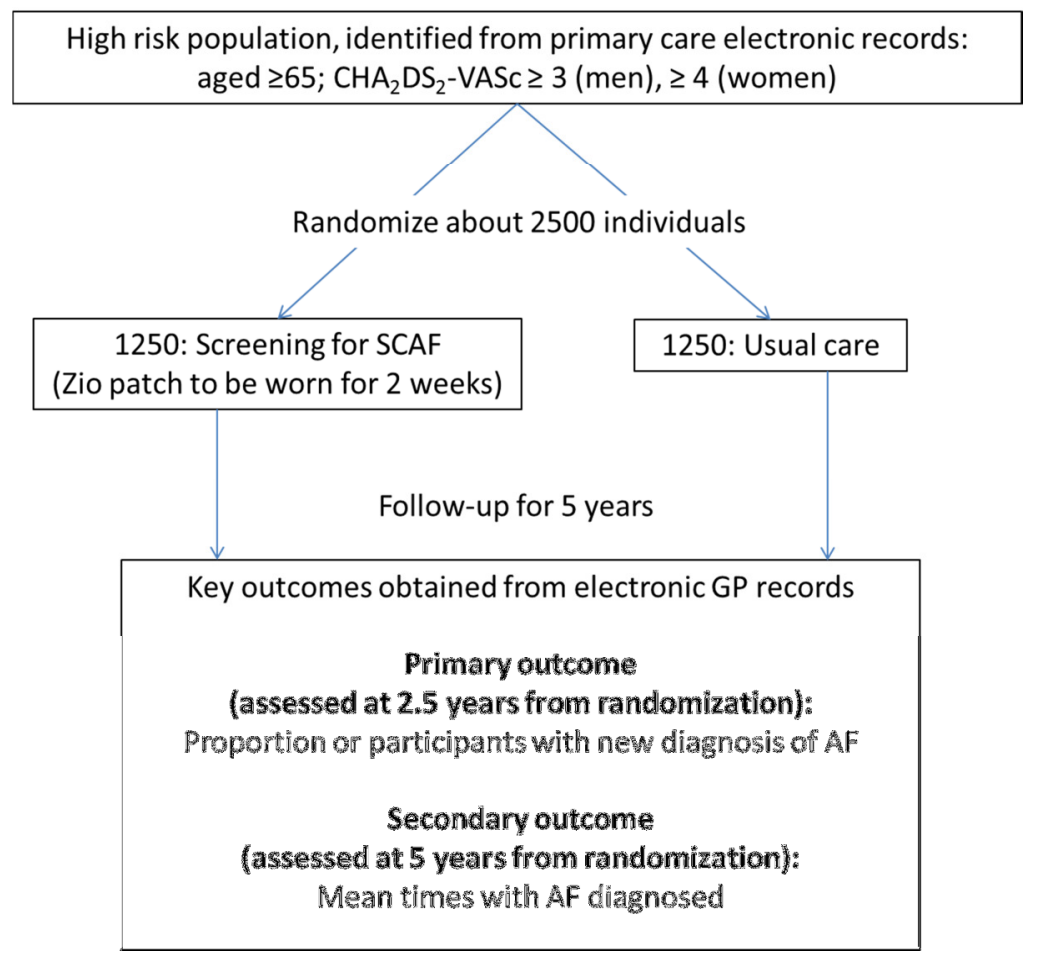

\section{PARTICIPANT IDENTIFICATION}

\subsection{Study Participants}

Participants are eligible for randomization if they satisfy all the inclusion and exclusion criteria and their GP is agreeable to their participation.

\subsection{Inclusion Criteria}

- Male or Female, aged $\geq 65$ years old.

- $\mathrm{CHA}_{2} \mathrm{DS}_{2}$-VASc score $\geq 3$ in men or $\geq 4$ in women (see Appendix 1)

- Participant is willing and able to give informed consent for participation in the study.

\subsection{Exclusion Criteria}

The participant may not enter the study if ANY of the following apply:

- Known to have AF or atrial flutter

- Latex allergy (given the potential for an allergic reaction to the Zio Patch).

\section{STUDY PROCEDURES}




\subsection{Recruitment}

GP practices (identified via local Clinical Research Networks and by direct approach) will identify eligible patients by running a predetermined electronic search of the practice database using the relevant local primary care software e.g. EMIS, SystemOne. An invitation letter, including an information leaflet, consent form, and Freepost return envelope, will then be sent by post (or, if practical, electronically via email) by the GP. Web-based and written study information will be available to provide potential participants with further information to help them make an informed decision as to whether to participate. A 24-hour Freephone service (established by CTSU for other large heart disease trials) will be available for study participants, their carers or their doctors to discuss any aspects of the study with experienced clinical staff at the coordinating centre.

\subsection{Enrolment and Eligibility Assessment}

There will be no face to face confirmation of eligibility. Participants will be asked to complete a baseline randomization questionnaire at the time of enrolment. This will include basic demographic information as well as medical information to confirm baseline $\mathrm{CHA}_{2} \mathrm{DS}_{2}$-VASc score (e.g. history of hypertension, stroke or diabetes, see Appendix 1).

\subsection{Informed Consent}

All participants will be required to provide written informed consent by returning a completed consent form to the coordinating centre. Information for participants regarding the study will be available on the study website and in written form. There will be the option for telephone or email enquiries to be made to the study team for any questions prior to enrolment. It will be clearly stated that the participant is free to withdraw from the study at any time for any reason without prejudice to future care, without affecting their legal rights, and with no obligation to give the reason for withdrawal. The participant will be allowed as much time as wished to consider the information, and the opportunity to question the study team, their GP or other independent parties to decide whether they will participate in the study. The original signed consent form will be retained at the central coordinating office and a copy will be returned to all participants who subsequently go on to be randomized into the trial.

\subsection{Randomization, blinding and code-breaking}

Participants who indicate on the randomization questionnaire that they are eligible and willing to join the trial, and provide written consent will be randomized by the central computer in CTSU, using a minimisation algorithm to ensure balance by important baseline variables. ${ }^{30}$

Eligible patients will be randomized in a 1:1 ratio to a single 2-week ECG monitoring period with the Zio Patch versus usual care. The study will not employ a sham-patch and neither the trial team nor the participants will be blinded to the intervention. All participants will receive a letter informing them which group them have been assigned to. The participant's GP will be notified that the participant has been randomized and the study arm; a copy of the participant's consent form (signature page) will also be included for reference. For all participants screened with the Zio Patch, the ECG findings will be reported back to the participant's GP and future management of any abnormal findings will be at the GPs discretion.

\subsection{Zio Patch administration}


For those participants randomized to receive the Zio Patch, the device will be posted to their home address with instructions on how to apply and care for it. Email and Freephone telephone advice lines will be available for those who need support or encounter problems.

Participants will be asked to wear the Zio Patch continuously for 2 weeks. A return envelope will be provided so that, once the two week period is completed, participants can post back the device for analysis. If participants do not return the device, efforts will be made by the research team to contact them to encourage them to return the device.

\subsection{Discontinuation/Withdrawal of Participants from Study}

Each participant has the right to withdraw from the study at any time, which will be made clear during the consent process. Any withdrawals will be recorded in the study database, including, where available, the reason for doing so.

\subsection{Definition of End of Study}

The end of study is defined as the date of the last electronic data download (from either central registries (NHS Digital) or from a participating GP practice. For the primary comparison, data will be sought at 2.5 years after randomization. For the secondary outcome further data will be sought at 5 years after randomization. However it is intended to continue to request longerterm data from NHS Digital (for up to 25 years after randomization) in order to undertake longer-term exploratory assessments of the clinical impact of allocation to screening (see Section 8.4).

\section{STATISTICS AND ANALYSIS}

\subsection{Initial assumptions}

Study duration: Logically, the length of the study should be the proposed time between screenings if this were put into practice. A follow-up period of 25 years is therefore proposed in AMALFI to allow for further research.

AF detection with usual care (opportunistic screening): The rate of opportunistic detection of AF in this population is unclear. Data from a 2014/2015 screening initiative across Dorset Clinical Commissioning Group reported opportunistic detection of SCAF in $0.7 \%$ of 61,000 people over the age of 65 who had a one-off pulse check while attending a flu clinic. ${ }^{31}$ However the proportion of attendees receiving a pulse check varied considerably between practices, ranging from $8 \%$ to $78 \%$ of individuals, with an average of $51 \%$. So, among all those aged $\geq 65$ years old SCAF may only be detected opportunistically in about $0.3-0.4 \%$ per annum.

The AMALFI population (with elevated $\mathrm{CHA}_{2} \mathrm{DS}_{2}$-VASc score) will tend to be at somewhat higher risk of SCAF, and so we anticipate that usual care with opportunistic screening by the GP may identify $A F$ in about $0.7 \%$ per annum.

AF detection with screening: The incidence of SCAF detection by screening with Zio Patch is unknown in this high risk population. Among 7000 people aged 75 screened with intermittent ECGs over a 2-week period, the rate of detection of SCAF was about $3 \% .{ }^{14}$ However, in AMALFI the monitoring will be continuous for two weeks (likely to provide higher rates of detection). 
Taking into account a moderate level of non-compliance with the patch, we estimate that a twoweek screening with Zio Patch may lead to a diagnosis of AF in around 3.75\% participants. Details of the assumptions and modelling of the rates for the purposes of the statistical power calculations can be found in appendix 2 .

AF detection after the screening period: In the screening group it is anticipated that further cases will continue to be identified by usual care after screening. These rates are assumed to increase with time from screening to converge with the rate found by usual care $10.7 \%$ per annum) and range from $0.2 \%$ during the first year following randomization, to $0.5 \%$ during the fifth year (see Appendix 2).

\subsection{Sample size and predicted number of diagnoses}

Given the assumptions above, the expected prevalence of AF over time in the AMALFI trial is shown in Figure 2 (see Appendix 2 for statistical model and calculations).

\section{Figure 2: Estimated cumulative percent of participants diagnosed with AF}

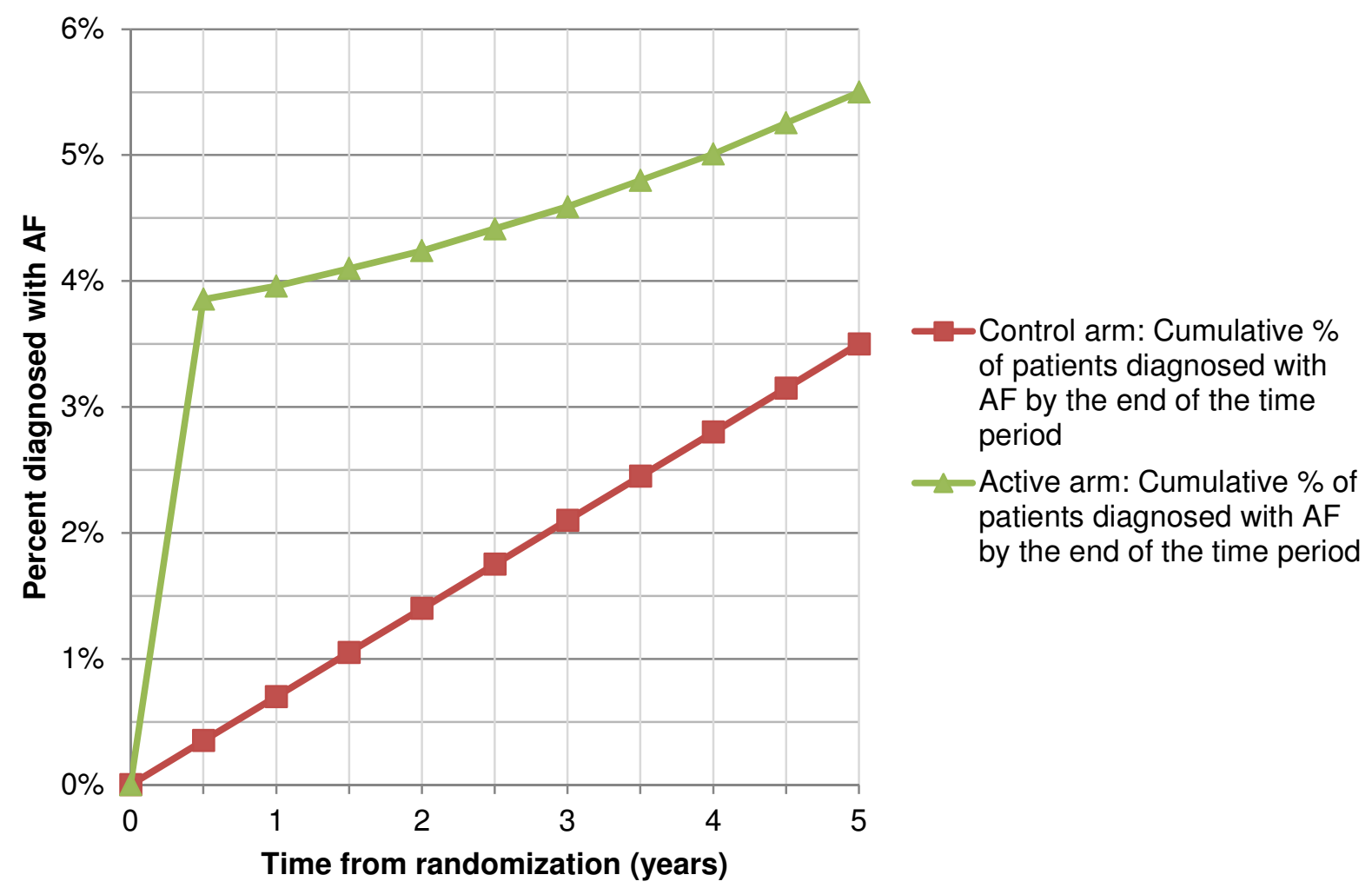

A sample size of 1250 per arm would give more than $90 \%$ power to detect the estimated difference in proportion with a diagnosis of $A F$ at 2.5 years of $1.75 \%$ versus $4.4 \%$ at $2 p<0.05$ (see Appendix 2). It is intended that compliance with the Zio Patch and rates of detection of AF will be monitored during the trial and, if they are substantially lower than anticipated, the Investigators will have the option to increase the sample size.

\subsection{Analysis of Outcome measures}

The fundamental assessments of efficacy will involve an "intention-to-treat" analysis among all randomized patients of allocation to screening versus allocation to usual care, irrespective of 
compliance. Analysis of the primary outcome: The chi-square test will be used to compare the proportion of participants diagnosed with AF at 2.5 years of follow up in all those randomized to screening versus all those randomized to usual care.

Analysis of the secondary outcome: Mean durations of time with AF diagnosed after 5 years of follow-up from randomization in all those randomized to screening compared to all those randomized to usual care will be compared using a permutation test to assess statistical significance. Those with no diagnosis of AF by the end of the study will contribute zero time with AF.

\subsection{Exploratory (non-randomized) assessments}

With only about 250 cases of AF expected during the 5-year period of the trial, a randomized comparison of the effects of screening for SCAF on the prevention of AF-related morbidity and mortality would have very low statistical power. However, in order to explore the potential clinical impact of screening we will perform an exploratory retrospective non-randomized comparison of rates of anticoagulation and of hospitalization (inpatient and outpatient) among those who were diagnosed with AF/SCAF by the end of follow-up according to whether or not they were diagnosed by Zio Patch screening or by usual care. It is recognised that such nonrandomized comparisons have considerable limitations. However it is hypothesised that the mode of diagnosis of AF may influence future management decisions (for example the decision whether to anticoagulate or not, or to refer to secondary care or not). These non-randomized comparisons may be informative in exploring such potential clinical management biases.

After the end of the scheduled study period, information about hospitalizations will continue to be collected from NHS Digital on all surviving participants (unless they have withdrawn consent) for up to an additional 20 years. These data will be used for longer-term exploratory assessments of the clinical impact of screening.

\section{DATA MANAGEMENT}

\subsection{Access to Data}

Direct access will be granted to authorised representatives from the Sponsor and host institution for monitoring and/or audit of the study to ensure compliance with regulations.

\subsection{Data Recording and Record Keeping}

Personal data will be entered electronically on a CTSU central restricted database that is password protected and following an operating procedure for data handling. Access to the data is granted on a 'need-to-know' basis, authorised by the study managers. All personal identifiers will be strongly encrypted and each participant allocated a study number at the point of enrolment. It will be possible to check back against the initial study number allocation to identify individual participants when necessary in the case of safety reasons or when needing to contact practices with results or request follow-up data.

All data will be held securely on computer servers managed by the University of Oxford, analysed by appropriately qualified staff with access restricted as appropriate for their role in the study, and used only for the medical research purposes outlined in this protocol. The data 
will be held for at least 25 years after the completion of the study and only used for the AMALFI study.

\section{ETHICAL AND REGULATORY CONSIDERATIONS}

The Chief Investigator will ensure that this study is conducted in accordance with the principles of the Declaration of Helsinki, and of Good Clinical Practice and in accordance with relevant regulations. The protocol, informed consent form, participant information sheet and any proposed advertising material (including all relevant substantial amendments to the original approved documents) will be submitted to the Health Research Authority (HRA) for written approval. The Chief Investigator shall submit once a year throughout the study, or on request, an Annual Progress report to the REC Committee, HRA (where required) host organisation and Sponsor. In addition, an End of Study notification and final report will be submitted to the same parties.

\section{FINANCE AND INSURANCE}

The study is funded by the National Institute for Health Research (NIHR) Oxford Biomedical Research Centre (BRC). iRhythm is providing additional support by providing the Zio Patches and reporting service free of charge during the AMALFI study. The University has a specialist insurance policy in place which would operate in the event of any participant suffering harm as a result of their involvement in the research (Newline Underwriting Management Ltd, at Lloyd's of London).

\section{PUBLICATION POLICY}

The Investigators (see page 1) will be involved in reviewing drafts of the manuscripts, abstracts, press releases and any other publications arising from the study. Authors will acknowledge that the study was funded by the National Institute for Health Research (NIHR) Oxford Biomedical Research Centre (BRC). Authorship will be determined in accordance with the ICMJE guidelines and other contributors will be acknowledged. 


\section{Appendix 1: Calculation of the $\mathrm{CHA}_{2} \mathrm{DS}_{2}$-VASc score}

\begin{tabular}{|c|c|c|}
\hline & Condition & Points \\
\hline C & Congestive heart failure (or Left ventricular systolic dysfunction) & 1 \\
\hline $\mathrm{H}$ & $\begin{array}{l}\text { Hypertension: blood pressure consistently above } 140 / 90 \mathrm{mmHg} \\
\text { (or treated hypertension on medication) }\end{array}$ & 1 \\
\hline$A_{2}$ & Age $\geq 75$ years & 2 \\
\hline $\mathrm{D}$ & Diabetes Mellitus & 1 \\
\hline $\mathrm{S}_{2}$ & Prior Stroke or TIA or thromboembolism & 2 \\
\hline V & $\begin{array}{l}\text { Vascular disease (e.g. peripheral artery disease, myocardial } \\
\text { infarction, aortic plaque) }\end{array}$ & 1 \\
\hline A & Age $65-74$ years & 1 \\
\hline Sc & Sex category (i.e. female sex) & 1 \\
\hline
\end{tabular}




\section{Appendix 2: Modelling of expected prevalence of AF over time and statistical power calculations}

\section{Primary outcome considerations:}

Logically, the length of study should be the proposed time between screenings if put into practice (i.e. potentially 5 years). The ideal primary outcome would be the time with AF diagnosed within 5 years of randomization, with those not getting AF having a time of zero with AF. The rationale is that this represents the window of opportunity for intervention. However, a more pragmatic alternative outcome, which allows for a more timely assessment of the value of screening with the Zio Patch, would be the proportion with AF around the middle of the study. The areas under the curves shown in Figure 2 (see protocol Section 8) give the expected mean times spent with $A F$ and are mostly driven by the proportions with $A F$ in the middle of the trial. Hence the proportion of participants with AF at 2.5 years has been chosen as the primary outcome for AMALFI.

\section{Assumptions for Figure 2 (see protocol Section 8):}

The probability of being diagnosed with AF is a time dependent Poisson process with a parameter slightly increasing with time, a+bt. Additionally, we assume that if someone would have had AF diagnosed at time $t$, there is a decreasing probability $\mathrm{e}+\mathrm{ft}$ that it would be preempted by patch related early detection of AF. We then chose a and b so that the AF diagnosis rates during years 1 and 2 were both $0.7 \%$ per annum, the anticipated detection rate with usual care.

e and $f$ were chosen to allow for non-compliance and to give decreasing probabilities over time of AFs that would have been diagnosed during a time period being pre-emptively detected by patch $(70 \%$ in the first year, $60 \%$ in the second year, $50 \%$ in the third, $40 \%$ in the fourth and $30 \%$ in the fifth year). An additional probability of $2 \%$ was assumed for patch related early detection of AF when no AF would have been detected within 5 years. It is assumed that patch related early detection of AF occurs evenly over the first 6 months from randomization. Table 1 below gives the estimated cumulative \% of participants being diagnosed with AF at the end of each year, based on this model and the assumptions described in Section 8 of the protocol.

\section{Sample size calculation:}

Table 2 below gives a set of power calculations based on the expected proportions diagnosed with AF at half yearly points up to 5 years. It is assumed that participants are randomized 1:1 to give to equal numbers in the control and active arms. The 2.5 year row is highlighted as being a suitable mid-study comparison time point for a proposed screening interval of 5 years. 
Table 1

\begin{tabular}{|c|c|c|c|c|c|c|c|}
\hline & \multicolumn{2}{|c|}{ Placebo arm } & \multicolumn{5}{|c|}{ Active arm } \\
\hline $\begin{array}{l}\text { Year } \\
\text { end }\end{array}$ & $\begin{array}{l}\text { \% of patients } \\
\text { diagnosed } \\
\text { with AF } \\
\text { within the } \\
\text { year }\end{array}$ & $\begin{array}{l}\text { Cumulative \% } \\
\text { of patients } \\
\text { diagnosed } \\
\text { with AF by } \\
\text { end of year }\end{array}$ & $\begin{array}{l}\% \text { of patients who } \\
\text { would have been } \\
\text { found to have AF in } \\
\text { year i, but found up } \\
\text { front by Zio Patch }\end{array}$ & $\begin{array}{c}\% \text { of } \\
\text { patients } \\
\text { diagnosed } \\
\text { by Zio } \\
\text { Patch }\end{array}$ & $\begin{array}{l}\text { Additional \% of } \\
\text { patients found to } \\
\text { have AF within the } \\
\text { year, but not with } \\
\text { Zio Patch }\end{array}$ & $\begin{array}{l}\% \text { of patients diagnosed } \\
\text { with AF within the year } \\
\text { (including } 2 \% \text { extra found } \\
\text { by Zio Patch, who } \\
\text { wouldn't have been } \\
\text { diagnosed with AF during } \\
\text { years 1-5) }\end{array}$ & $\begin{array}{l}\text { Cumulative \% } \\
\text { of patients } \\
\text { diagnosed } \\
\text { with AF by } \\
\text { end of year }\end{array}$ \\
\hline 1 & $0.70 \%$ & $0.70 \%$ & $70 \%$ & $0.49 \%$ & $0.21 \%$ & $3.96 \%$ & $3.96 \%$ \\
\hline 2 & $0.70 \%$ & $1.40 \%$ & $60 \%$ & $0.42 \%$ & $0.28 \%$ & $0.28 \%$ & $4.24 \%$ \\
\hline 3 & $0.70 \%$ & $2.10 \%$ & $50 \%$ & $0.35 \%$ & $0.35 \%$ & $0.35 \%$ & $4.59 \%$ \\
\hline 4 & $0.70 \%$ & $2.80 \%$ & $40 \%$ & $0.28 \%$ & $0.42 \%$ & $0.42 \%$ & $5.01 \%$ \\
\hline 5 & $0.70 \%$ & $3.50 \%$ & $30 \%$ & $0.21 \%$ & $0.49 \%$ & $0.49 \%$ & $5.50 \%$ \\
\hline
\end{tabular}

Table 2

\begin{tabular}{|c|c|c|c|c|c|}
\hline \multirow{2}{*}{$\begin{array}{c}\text { Comparison } \\
\text { time point }\end{array}$} & \multicolumn{2}{|c|}{$\begin{array}{c}\text { Expected proportion with } \\
\text { AF at time point }\end{array}$} & \multirow{2}{*}{ Alpha } & Power & $\begin{array}{c}\text { Total sample size } \\
\text { (continuity corrected) }\end{array}$ \\
\cline { 2 - 5 } & Control arm & Active arm & & & 1019 \\
\hline 1 & $0.70 \%$ & $3.96 \%$ & 0.05 & $90 \%$ & 1261 \\
\hline 1.5 & $1.05 \%$ & $4.10 \%$ & 0.05 & $90 \%$ & 1565 \\
\hline 2 & $1.40 \%$ & $4.24 \%$ & 0.05 & $90 \%$ & 1914 \\
\hline 2.5 & $1.75 \%$ & $4.41 \%$ & 0.05 & $90 \%$ & 2348 \\
\hline 3 & $2.10 \%$ & $4.59 \%$ & 0.05 & $90 \%$ & 2825 \\
\hline 3.5 & $2.45 \%$ & $4.80 \%$ & 0.05 & $90 \%$ & 3406 \\
\hline 4 & $2.80 \%$ & $5.01 \%$ & 0.05 & $90 \%$ & 4004 \\
\hline 4.5 & $3.15 \%$ & $5.25 \%$ & 0.05 & $90 \%$ & 4711 \\
\hline 5 & $3.50 \%$ & $5.50 \%$ & 0.05 & $90 \%$ & \\
\hline
\end{tabular}

Clinical Research Protocol Template version 13.0

C Copyright: The University of Oxford and Oxford University Hospitals NHS Foundation Trust 2016 


\section{REFERENCES}

1. Chugh, S.S., et al. Worldwide epidemiology of atrial fibrillation: a Global Burden of Disease 2010 Study. Circulation 129, 837-847 (2014).

2. Miyasaka, Y., et al. Secular trends in incidence of atrial fibrillation in Olmsted County, Minnesota, 1980 to 2000, and implications on the projections for future prevalence. Circulation 114, 119-125 (2006).

3. Krijthe, B.P., et al. Projections on the number of individuals with atrial fibrillation in the European Union, from 2000 to 2060. Eur Heart J 34, 2746-2751 (2013).

4. Ball, J., Carrington, M.J., McMurray, J.J. \& Stewart, S. Atrial fibrillation: profile and burden of an evolving epidemic in the 21st century. International journal of cardiology 167, 1807-1824 (2013).

5. Stewart, S., Murphy, N.F., Walker, A., McGuire, A. \& McMurray, J.J. Cost of an emerging epidemic: an economic analysis of atrial fibrillation in the UK. Heart 90, 286-292 (2004).

6. Freedman, B., et al. Screening for Atrial Fibrillation: A Report of the AF-SCREEN International Collaboration. Circulation 135, 1851-1867 (2017).

7. Glotzer, T.V. \& Ziegler, P.D. Cryptogenic stroke: Is silent atrial fibrillation the culprit? Heart Rhythm 12, 234-241 (2015).

8. Sacco, R.L., et al. Infarcts of undetermined cause: the NINCDS Stroke Data Bank. Annals of neurology 25, 382-390 (1989).

9. Gladstone, D.J., et al. Atrial fibrillation in patients with cryptogenic stroke. $N$ Engl J Med 370, 2467-2477 (2014).

10. Sanna, T., Diener, H., Passman, R. \& etal. Cryptogenic Stroke and Underlying Atrial Fibrillation; from the CRYSTAL AF Investigators. N Engl J Med 370, 2478-2486 (2014).

11. Healey, J.S., et al. Subclinical Atrial Fibrillation in Older Patients. Circulation (2017).

12. Brambatti, M., et al. Temporal relationship between subclinical atrial fibrillation and embolic events. Circulation 129, 2094-2099 (2014).

13. Lowres, N., Neubeck, L., Redfern, J. \& Freedman, S. Screening to identify unknown atrial fibrillation. A systematic review. Thromb Haemost 110, 213-222 (2013).

14. Svennberg, E., et al. Mass Screening for Untreated Atrial Fibrillation: The STROKESTOP Study. Circulation 131, 2176-2184 (2015).

15. Lamassa, M., et al. Characteristics, outcome, and care of stroke associated with atrial fibrillation in Europe: data from a multicenter multinational hospital-based registry (The European Community Stroke Project). Stroke 32, 392-398 (2001).

16. Hart, R., Sherman, D., Easton, J. \& Cairns, J. Prevention of stroke in patients with nonvalvular atrial fibrillation. Neurology 51, 674-681 (1998).

17. Xiong, Q., Proietti, M., Senoo, K. \& Lip, G. Asymptomatic versus symptomatic atrial fibrillation: A systematic review of age/gender differences and cardiovascular outcomes. Int J Cardiol. 191, 172-177 (2015).

18. Hohnloser, S., et al. ASymptomatic atrial fibrillation and Stroke Evaluation in pacemaker patients and the atrial fibrillation Reduction atrial pacing Trial (ASSERT). Am Heart J 152, 442-447 (2006).

19. Van Gelder, I.C., et al. Duration of device-detected subclinical atrial fibrillation and occurrence of stroke in ASSERT. Eur Heart J 38, 1339-1344 (2017).

20. Wilson, J. \& Jungner, G. Principles and practice of screening for disease. . (ed. 34, P.H.P.N.) (WHO, Geneva, 1968). 
21. Arun, C., Al-Bermani, A., Stannard, K. \& Taylor, R. Long-term impact of retinal screening on significant diabetes-related visual impairment in the working age population. Diabetic Medicine 26, 489-492 (2009 Apr).

22. Misra, A., et al. Trends in yield and effects of screening intervals during 17 years of a large community-based diabetic retinopathy screening programme. Diabetic Medicine 26, 1040-1047 (2009).

23. Moran, P., Teljeur, C., Ryan, M. \& Smith, S. Systematic screening for the detection of atrial fibrillation. Cochrane Database of Systematic Reviews (2016).

24. Fitzmaurice, D., et al. Screening versus routine practice in detection of atrial fibrillation in patients aged 65 or over: cluster randomised controlled trial. BMJ 335, 383 (2007).

25. Halcox, J.P.J., et al. Assessment of Remote Heart Rhythm Sampling Using the AliveCor Heart Monitor to Screen for Atrial Fibrillation: The REHEARSE-AF Study. Circulation 136, 1784-1794 (2017).

26. NICE. Zio Service for detecting cardiac arrhythmias. Medtech innovation briefing [MIB101] Published date: March 2017. (ed. Excellence, N.I.f.H.a.C.) (https://www.nice.org.uk/advice/mib101, 2017).

27. January, C.T., et al. 2014 AHA/ACC/HRS guideline for the management of patients with atrial fibrillation: a report of the American College of Cardiology/American Heart Association Task Force on Practice Guidelines and the Heart Rhythm Society. Journal of the American College of Cardiology 64, e1-76 (2014).

28. Kirchhof, P., et al. 2016 ESC Guidelines for the management of atrial fibrillation developed in collaboration with EACTS. Eur Heart J 37, 2893-2962 (2016).

29. Engel, J., et al. Estimation of patient compliance in application of adherent mobile cardiac telemetry device. Conf Proc IEEE Eng Med Biol Soc. 2011, 1536-1539 (2011).

30. Pocock, S.J. \& Simon, R. Sequential treatment assignment with balancing for prognostic factors in the controlled clinical trial. Biometrics 31, 103-115 (1975).

31. Arathoon, N. Opportunistic screening for Atrial Fibrillation for over 65 year olds during flu clinics and anticoagulation for the prevention of stroke in primary care. (ed. Group, D.C.C.) (http://www.londonscn.nhs.uk/wp-content/uploads/2017/07/dorset-ccg.pdf, 2015). 